\title{
Spin susceptibility and polarization field in a dilute two-dimensional electron system in (111) silicon
}

\author{
A. A. Kapustin, A. A. Shashkin, and V. T. Dolgopolov \\ Institute of Solid State Physics, Chernogolovka, Moscow District 142432, Russia \\ M. Goiran and H. Rakoto* \\ CNRS, LNCMP, 143 Avenue de Rangueil, F-31400 Toulouse, France \\ Universite de Toulouse, UPS, INSA, LNCMP, F-31077 Toulouse, France \\ Z. D. Kvon \\ Institute of Semiconductor Physics, Novosibirsk, 630090, Russia
}

\begin{abstract}
We find that the polarization field, $B_{\chi}$, obtained by scaling the weak-parallel-field magnetoresistance at different electron densities in a dilute two-dimensional electron system in (111) silicon, corresponds to the spin susceptibility that grows strongly at low densities. The polarization field, $B_{\text {sat }}$, determined by resistance saturation, turns out to deviate to lower values than $B_{\chi}$ with increasing electron density, which can be explained by filling of the upper electron subbands in the fully spin-polarized regime.
\end{abstract}

PACS numbers: 71.30.+h,73.40.Qv,71.18.+y

Much interest has been attracted recently to the anomalous properties of strongly interacting twodimensional (2D) electron systems. The interaction strength is characterized by the Wigner-Seitz radius, $r_{s}=1 /\left(\pi n_{s}\right)^{1 / 2} a_{B}$ (where $n_{s}$ is the electron density and $a_{B}$ is the effective Bohr radius in semiconductor), which is equal in the single-valley case to the ratio of the Coulomb and the Fermi energies. While at high electron densities $\left(r_{s}<1\right)$ conventional Fermi-liquid behavior is established, at very low electron densities $\left(r_{s} \gg 1\right)$ formation of the Wigner crystal is expected [1, 2, 3]. It was not until recently that qualitative deviations from the weakly-interacting Fermi liquid behavior (in particular, the drastic increase of the effective electron mass with decreasing electron density) have been found in strongly correlated 2D electron systems $\left(r_{s} \gtrsim 10\right)$ [4, [5, 6].

The strongest many-body effects have been observed in (100)-silicon metal-oxide-semiconductor field-effect transistors (MOSFETs). This has particularly stimulated a new series of experiments in (111)-silicon MOSFETs [7, 8, 9, 10]. It was found in Ref. [10] by an analysis of the temperature-dependent Shubnikov-de Haas oscillations that the strongly increased mass is also the case in a dilute $2 \mathrm{D}$ electron system in (111) silicon with relatively high disorder, as indicated by both the absence of metallic temperature dependence of zero-field resistance and the relatively low mobility. Moreover, it was found that the relative mass enhancement as a function of $r_{s}$ is system- and disorder-independent being determined by electron-electron interactions only. However, no sign of the enhanced spin susceptibility, $\chi$, which is proportional to the product of the $g$ factor and effective mass, $m$, was observed in parallel-field magnetoresistance measurements [7]. Rather than tending to zero at a finite electron density like in (100)-silicon MOSFETs [4], the resistance saturation field, $B_{\text {sat }}$, corresponding to the onset of full polarization of the electrons' spins was found to increase approximately proportionally with the electron density. It is worth noting that the valley degeneracy in (111)-silicon MOSFETs is equal to $g_{v}=2$ (as well as the spin degeneracy $g_{s}=2$ ), rather than $g_{v}=6$ (see, e.g., Ref. [11]), as inferred from the filling factor change for the period of Shubnikov-de Haas oscillations in weak magnetic fields, equal to 4 . The reduced valley degeneracy was attributed, in particular, to the existence of inhomogeneous strains at the interface [12]. The upper electron subbands can be important for the behavior of $B_{\text {sat }}$. Filling of these subbands has been observed recently in studies of Shubnikov-de Haas oscillations in (111)-silicon samples of a special design: a 2D electron system forms at the interface between (111) silicon and vacuum [9].

In this paper, we study low-temperature parallel-field magnetotransport in a 2D electron system in (111) silicon in a wide range of electron densities. We find that the polarization magnetic field, $B_{\chi}$, obtained by scaling the weak-parallel-field magnetoresistance at different electron densities, tends to zero at a finite electron density in a linear fashion. It corresponds to the spin susceptibility $\chi \propto g m$ that increases strongly at low densities, $B_{\chi} \propto n_{s} / \chi$, which is consistent with the increase of $m$ observed earlier in this electron system. The polarization field $B_{\text {sat }}$, determined by resistance saturation, turns out to deviate to lower values than $B_{\chi}$ with increasing electron density. This can be explained by the parallel-field-induced filling of the upper electron subbands corresponding to another two valleys in (111) silicon. The subbands' splitting in our samples is estimated at $\Delta \approx 2 \mathrm{meV}$.

Measurements were made in an Oxford dilution refrigerator with a base temperature of $\approx 30 \mathrm{mK}$ (Chernogolovka) and a dilution refrigerator with a base 
temperature of $\approx 80 \mathrm{mK}$ (Toulouse) in magnetic fields up to $14 \mathrm{~T}$ on (111)-silicon MOSFETs similar to those previously used in Ref. 7]. Samples had the Hall bar geometry with width $400 \mu \mathrm{m}$ equal to the distance between the potential probes. Application of a dc voltage to the gate relative to the contacts allowed one to control the electron density. Oxide thickness was equal to $154 \mathrm{~nm}$. In highest-mobility samples, the normal of the sample surface was tilted from [111]- toward [110]-direction by a small angle of $8^{\circ}$. Anisotropy for electron transport in such samples does not exceed $5 \%$ at $n_{s}=3 \times 10^{11} \mathrm{~cm}^{-2}$ and increases weakly with electron density, staying below $25 \%$ at $n_{s}=3 \times 10^{12} \mathrm{~cm}^{-2}[10]$. The resistance was measured by a standard 4-terminal technique at a low frequency $(5-10 \mathrm{~Hz})$ to minimize the out-of-phase signal. Excitation current was kept low enough (below $2 \mathrm{nA}$ ) to ensure that measurements were taken in the linear regime of response. The current was parallel to the inplane magnetic field. Electron densities were determined by Shubnikov-de Haas oscillations in perpendicular magnetic fields, for which the sample orientation was changed at room temperature and another run was made. The threshold voltage was checked to be the same in different runs, although the peak electron mobility at $T \approx 1.5 \mathrm{~K}$ varied in the range between 2500 and $4000 \mathrm{~cm}^{2} / \mathrm{Vs}$. Two samples were used in the experiments; below, we show data obtained on one of them. The results reported are independent of a sample and a sample state and are reproducible in different cryostats.

Additional measurements were made in a rotatorequipped $\mathrm{He}^{4}$ cryostat at a temperature of $\approx 1.5 \mathrm{~K}$ in pulsed magnetic fields (Toulouse). In each pulse, the magnetic field swept up to $\approx 48 \mathrm{~T}$ (or lower) with rising and falling times of about $50 \mathrm{~ms}$ and $0.3 \mathrm{~s}$, respectively. Excitation current with frequency $2 \mathrm{kHz}$ did not exceed $600 \mathrm{nA}$.

Typical curves of the low-temperature magnetoresistance $\rho(B)$ in a parallel magnetic field are displayed in Fig. 1(a). The resistivity increases with field and saturates above a certain density-dependent magnetic field. Deep in the metallic regime $\left(\rho \ll h / e^{2}\right)$, the magnetoresistance depends weakly on temperature in the experimental range of temperatures (at $T \leq 1.5 \mathrm{~K}$ ). This limit is realized for progressively narrower initial intervals on the curve $\rho(B)$, as the electron density is lowered. The data discussed in this paper are obtained deep in the metallic regime.

In Fig. 1(b), we show how the normalized weak-field magnetoresistance, measured at different electron densities, collapses onto a single curve when plotted as a function of $B / B_{\chi}$. The scaling parameter $B_{\chi}$ has been normalized to correspond to the magnetic field at which the magnetoresistance at the lowest electron densities in the good metallic regime saturates and full spin polarization of the electrons is reached [13, 14, 15]. Within the accuracy with which the saturation field $B_{\text {sat }}$ can be determined, we use for the normalization the curve $\rho(B)$ at $n_{s} \approx 6 \times 10^{11} \mathrm{~cm}^{-2}$, measured in pulsed magnetic fields;
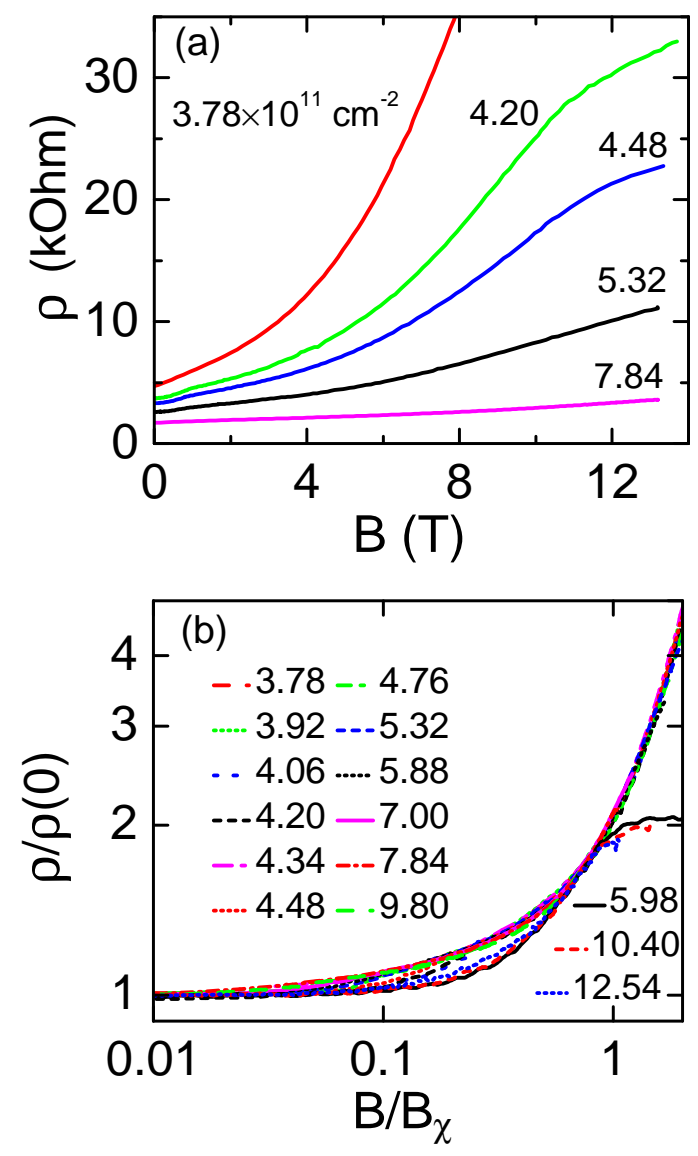

FIG. 1: (a) Magnetoresistance in dc parallel magnetic fields at a temperature of $\approx 80 \mathrm{mK}$ at different electron densities in the metallic regime. (b) Scaled curves of the normalized magnetoresistance at different $n_{s}$ versus $B / B_{\chi}$ for dc (upper set) and pulsed (lower set) parallel magnetic fields. The electron densities are indicated in units of $10^{11} \mathrm{~cm}^{-2}$.

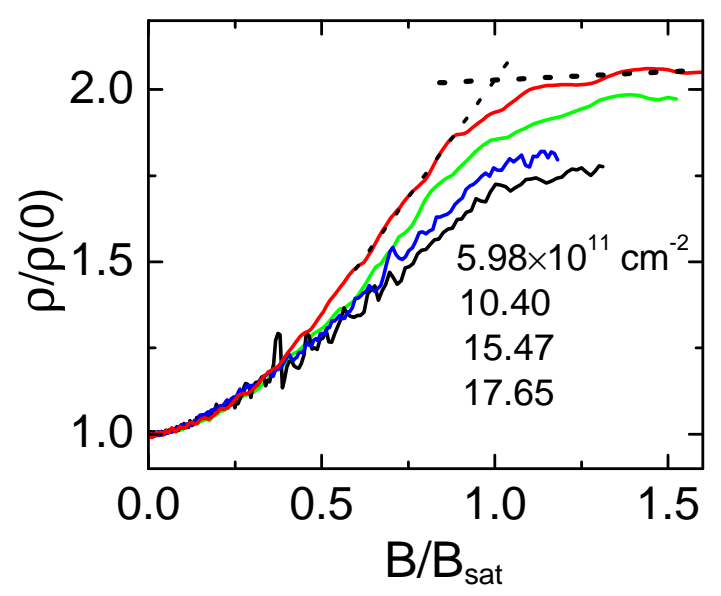

FIG. 2: The normalized magnetoresistance as a function of $B / B_{\text {sat }}$ at different electron densities for pulsed parallel magnetic fields. Also shown is the way to determine the saturation field. 


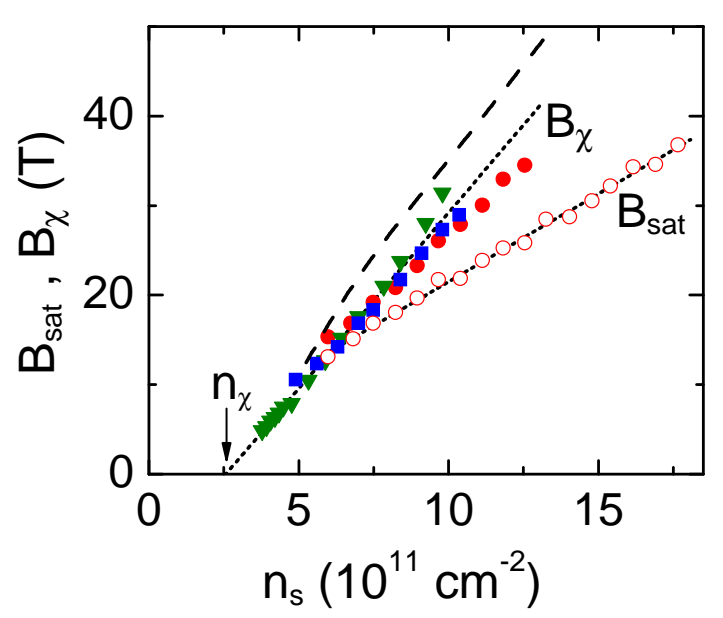

FIG. 3: Dependence of the fields $B_{\text {sat }}$ (open circles) and $B_{\chi}$ (squares, triangles, and dots) on electron density. Squares and triangles are obtained in two runs in dc magnetic fields, and dots and open circles correspond to pulsed magnetic fields. The dotted lines are linear fits. The fit for $B_{\chi}$ extrapolates to a critical density $n_{\chi}$. Also shown by a dashed line is the data for the enhanced effective mass of Ref. [10] recalculated into $B_{\chi}$ using the value of $g=2$ in bulk silicon.

we have verified that the fields $B_{\text {sat }}$ and $B_{\chi}$ are practically temperature-independent in the range of temperatures used. At high electron densities, the value of $B_{\text {sat }}$ is noticeably smaller than $B_{\chi}$ in this electron system, as is evident from Fig. 2 which shows the normalized magnetoresistance versus $B / B_{\text {sat }}$ in pulsed magnetic fields. Apparently, the scaled data in Fig. 1(b) are a function of the degree of spin polarization, $\xi=B / B_{\chi}$, in weak magnetic fields but not at $\xi \approx 1$.

In Fig. 3, we show the so-determined polarization fields $B_{\text {sat }}$ and $B_{\chi}$ as a function of electron density. The field $B_{\chi}$ obtained by scaling the weak-field magnetoresistance depends linearly on $n_{s}$ and tends to vanish at a finite electron density, $n_{\chi}$. Our procedure provides good accuracy for determining the behavior of $B_{\chi}$ with electron density, i.e., the functional form of $B_{\chi}\left(n_{s}\right)$, even though the absolute value of $B_{\chi}$ is determined not so accurately. Based on the form of the spin polarization parameter $\xi=\chi B / \mu_{B} n_{s}=g \mu_{B} B / 2 E_{F}=B / B_{\chi}$ (where $\mu_{B}$ is the Bohr magneton and $E_{F}=\pi \hbar^{2} n_{s} / 2 m$ is the Fermi energy in $B=0$ ), we have recalculated the data for the enhanced effective mass $m\left(n_{s}\right)$ of Ref. [10] into $B_{\chi}\left(n_{s}\right)$ (dashed line), using the value of $g=2$ in bulk silicon which is close to the $g$ factor measured in silicon MOSFETs [4]. Both dependences $B_{\chi}\left(n_{s}\right)$ are in agreement with each other, and the spin susceptibility $\chi \propto g m$ is strongly enhanced at low densities. The resistance saturation field $B_{\text {sat }}$ deviates to lower values than $B_{\chi}$ with increasing electron density, $B_{\text {sat }}\left(n_{s}\right)$ being a linear dependence with approximately twice as small a slope. We note that our data for $B_{\text {sat }}$ are concurrent with earlier results [7].

The observed behavior of the polarization field $B_{\chi}$ with

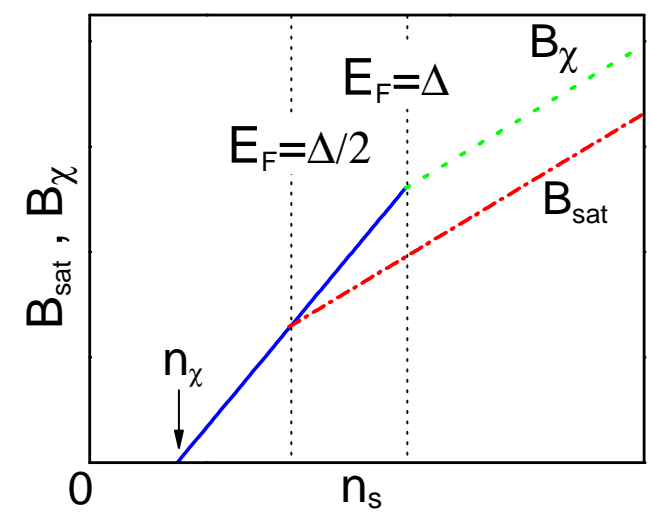

FIG. 4: The schematic behavior of the fields $B_{\text {sat }}$ and $B_{\chi}$ with electron density, as discussed in text. There are break points in the dependences $B_{\text {sat }}\left(n_{s}\right)$ at $E_{F}=\Delta / 2\left(\right.$ or $\left.E_{F}\left(B_{\text {sat }}\right)=\Delta\right)$ and $B_{\chi}\left(n_{s}\right)$ at $E_{F}=\Delta$.

electron density, which corresponds to the sharply increased spin susceptibility at low densities, is very similar to that in a 2D electron system in (100)-silicon MOSFETs [15]. The same interaction strength in (111)-silicon MOSFETs is expected to be achieved at electron densities about four times higher than in (100) silicon, since in the first case the effective mass $m_{b}=0.358 m_{e}$ (where $m_{e}$ is the free electron mass) in bulk silicon is approximately twice as large. Based on the critical density, $n_{\chi} \approx 8 \times 10^{10} \mathrm{~cm}^{-2}$, of vanishing polarization field for (100)-silicon MOSFETs, one expects the critical region in (111) silicon at electron densities about $3 \times 10^{11} \mathrm{~cm}^{-2}$. This is consistent with our results for $B_{\chi}$ (Fig. 3).

It is interesting that the polarization field $B_{\text {sat }}\left(n_{s}\right)$ behaves differently from $B_{\chi}\left(n_{s}\right)$. This finding is in contrast to the case of (100)-silicon MOSFETs where the fields $B_{\chi}$ and $B_{\text {sat }}$ are practically the same [4] and the scaled data are described reasonably well by the theoretical dependence of the normalized magnetoresistance on the degree of spin polarization [16] in a wide range of $\xi$. Clearly, the discrepancy between both polarization fields in (111)-silicon MOSFETs reflects the fact that the spin polarization parameter is not linear in $B$ at large $\xi \approx 1$. Below, we discuss possible mechanisms for the effect.

A simple reason for the value of saturation field to become reduced as the electron density is increased might be the orbital effects that occur when the thickness of the $2 \mathrm{D}$ electron system becomes comparable to the magnetic length, $l_{B}=(\hbar c / e B)^{1 / 2}$. These are expected to lead to an increase of the effective mass with parallel magnetic field (see, e.g., Ref. 17]). However, no such reduction in the $B_{\text {sat }}\left(n_{s}\right)$ dependence has been found for (100)-silicon MOSFETs in the same range of pulsed parallel magnetic fields [18]. Because the corresponding thicknesses of the 2D electron systems [19] for both orientations in silicon MOSFETs are close to each other, the orbital effects are likely to be small for our case.

Another reason for the observed reduction of $B_{\text {sat }}$ is the filling of the upper electron subbands in the fully 
spin-polarized regime. The Fermi energy should double at the onset of complete spin polarization [16], if $E_{F}$ is below the mid-gap, $\Delta / 2$ (here $\Delta$ is the subband separation). In the opposite case $E_{F}>\Delta / 2$ the parallel magnetic field promotes filling of the upper subbands. These are empty in the experimental range of $n_{s}$ in our samples in $B=0$ (i.e., $E_{F}<\Delta$ ) but can be filled at high electron densities in parallel magnetic fields provided the condition $E_{F}>\Delta / 2$ is met. In this case the Fermi energy at the onset of complete spin polarization, $E_{F}\left(B_{\text {sat }}\right)=g \mu_{B} B_{\text {sat }}$, exceeds the splitting $\Delta$ and should increase with electron density with a slope reduced by the factor of two (see Fig. 4), as can be expected from the results of Ref. [9]. There, the filling factor change for the period of the weak-field Shubnikov-de Haas oscillations in (111)-silicon samples has been found to be equal to 8, which immediately indicates that the upper subbands in question correspond to another two valleys. The slope reduction expected for both $E_{F}\left(B_{\text {sat }}\right)$ and $B_{\text {sat }}$ is in agreement with the data in Fig. 3. Note that the observed linear increase of $B_{\text {sat }}$ with $n_{s}$ indicates that a possible influence of the diamagnetic contribution to $\Delta$ is small. Thus, the parallel-field-induced filling of the upper subbands corresponding to another two valleys in (111) silicon can account for the experimental behavior of the saturation field.

We estimate the splitting of the subbands at $\Delta \approx$ $2 \mathrm{meV}$ for $n_{s} \approx 6 \times 10^{11} \mathrm{~cm}^{-2}$. This value is appreciably bigger than the splitting $\Delta \approx 0.6 \mathrm{meV}$ obtained in Ref. [9], which is consistent with the pattern of Shubnikov-de Haas oscillations in the two sets of samples. The fact that the splitting $\Delta$ is not universal in different samples may be due to different inhomogeneous strains at the interface [12] which are sensitive to a particular procedure of sample fabrication. Based on the experimental data and ignoring the possible (linear) dependence of $\Delta$ on $n_{s}$, one can expect filling of the upper subbands in $B=0$ at electron densities above $\approx 1.5 \times 10^{12} \mathrm{~cm}^{-2}$. Once no double the periodicity of Shubnikov-de Haas oscillations in weak magnetic fields was observed in our samples up to $n_{s} \approx 2.5 \times 10^{12} \mathrm{~cm}^{-2}$ [10], yet higher electron densities should be tried to clarify the point. Note that at very high electron densities about $1 \times 10^{13} \mathrm{~cm}^{-2}$, observation of the sixfold valley degeneracy was reported [20], although the high $g_{v}$ can be promoted by additional annealing of the samples [21].

In summary, we have found that the polarization field $B_{\chi}$, obtained by scaling the weak-parallel-field magnetoresistance at different electron densities in (111)-silicon MOSFETs, tends to zero at a finite electron density in a linear fashion. It corresponds to the spin susceptibility that increases strongly at low densities. This is consistent with the increase of the effective mass observed earlier in this electron system. The polarization field $B_{\text {sat }}$, determined by resistance saturation, turns out to deviate to lower values than $B_{\chi}$ with increasing electron density. The reduction of $B_{\text {sat }}$ can be explained by filling of the upper electron subbands in the fully spin-polarized regime. The results obtained allow us to estimate the subbands' splitting in our samples.

We gratefully acknowledge discussions with A. Gold and S. V. Kravchenko. We would like to thank M. Nardonne for technical assistance. This work was supported by the RFBR, RAS, and the Programme "The State Support of Leading Scientific Schools".
[*] Deceased.

[1] A. V. Chaplik, Sov. Phys. JETP 35, 395 (1972).

[2] B. Tanatar and D. M. Ceperley, Phys. Rev. B 39, 5005 (1989).

[3] C. Attaccalite, S. Moroni, P. Gori-Giorgi, and G. B. Bachelet, Phys. Rev. Lett. 88, 256601 (2002).

[4] S. V. Kravchenko and M. P. Sarachik, Rep. Prog. Phys. 67, 1 (2004); A. A. Shashkin, Phys. Usp. 48, 129 (2005).

[5] A. A. Shashkin, S. Anissimova, M. R. Sakr, S. V. Kravchenko, V. T. Dolgopolov, and T. M. Klapwijk, Phys. Rev. Lett. 96, 036403 (2006).

[6] S. Anissimova, A. Venkatesan, A. A. Shashkin, M. R. Sakr, S. V. Kravchenko, and T. M. Klapwijk, Phys. Rev. Lett. 96, 046409 (2006).

[7] O. Estibals, Z. D. Kvon, G. M. Gusev, G. Arnaud, and J. C. Portal, Physica E 22, 446 (2004).

[8] K. Eng, R. N. McFarland, and B. E. Kane, Appl. Phys. Lett. 87, 052106 (2005); Physica E 34, 701 (2006).

[9] K. Eng, R. N. McFarland, and B. E. Kane, Phys. Rev. Lett. 99, 016801 (2007).

[10] A. A. Shashkin, A. A. Kapustin, E. V. Deviatov, V. T. Dolgopolov, and Z. D. Kvon, Phys. Rev. B 76, 241302(R) (2007).
[11] T. Neugebauer, K. v. Klitzing, G. Landwehr, and G. Dorda, Solid State Commun. 17, 295 (1975).

[12] D. C. Tsui and G. Kaminsky, Solid State Commun. 20, 93 (1976).

[13] T. Okamoto, K. Hosoya, S. Kawaji, and A. Yagi, Phys. Rev. Lett. 82, 3875 (1999).

[14] S. A. Vitkalov, H. Zheng, K. M. Mertes, M. P. Sarachik, and T. M. Klapwijk, Phys. Rev. Lett. 85, 2164 (2000).

[15] A. A. Shashkin, S. V. Kravchenko, V. T. Dolgopolov, and T. M. Klapwijk, Phys. Rev. Lett. 87, 086801 (2001).

[16] V. T. Dolgopolov and A. Gold, JETP Lett. 71, 27 (2000); A. Gold and V. T. Dolgopolov, Physica E 17, 280 (2003).

[17] E. Tutuc, S. Melinte, E. P. De Poortere, M. Shayegan, and R. Winkler, Phys. Rev. B 67, 241309(R) (2003).

[18] J. M. Broto, M. Goiran, H. Rakoto, A. Gold, and V. T. Dolgopolov, Phys. Rev. B 67, 161304(R) (2003).

[19] T. Ando, A. B. Fowler, and F. Stern, Rev. Mod. Phys. 54, 437 (1982).

[20] D. C. Tsui and G. Kaminsky, Phys. Rev. Lett. 42, 595 (1979).

[21] T. Cole and B. D. McCombe, Phys. Rev. B 29, 3180 (1984). 